\title{
Voice Therapy in Muscle Tension Dysphonia Cases
}

\author{
${ }^{1}$ Sachender Pal Singh, ${ }^{2}$ Smrity Rupa Borah Dutta
}

\begin{abstract}
Muscle tension dysphonia (MTD) is a condition where phonation is associated with excessive muscular tension or muscle misuse. It has multifactorial etiologies. It can be a primary or secondary MTD. While it can affect anyone, sufferers usually belong to a particular group. It has very serious impact on sufferer's personal, social and professional life.

We are presenting here, our 20 months prospective study done in the department of otorhinolaryngology, Silchar Medical College and Hospital from June 2012 to July 2013.

Voice therapy was given to every patient, whether primary or secondary MTD. Pre-therapy vs post-therapy comparisons were made of self-ratings of voice handicap index, auditoryperceptual ratings as well as visual-perceptual evaluations of laryngeal images.

Outcome of voice therapy results (Graphs 1 and 2) in such patients were found to be very good. As the disease is multifactorial, treatment approach should be broad-based involving multidisciplinary team.
\end{abstract}

Keywords: Circumlaryngeal massage, Dysphonia plica ventricularis, GRABS score.

Abbreviations: Vocal Cord Nodule (N), Vocal Polyp (P), Laryngopharyngeal Reflux (LPR), Presbylaryngis (PL), Cut Throat injury (CT), Primary Muscle Tension Dysphonia (PMTD), Dysphonia Plica ventricular (DPV).

How to cite this article: Singh SP, Dutta SRB. Voice Therapy in Muscle Tension Dysphonia Cases. Int J Phonosurg Laryngol 2015;5(1):20-24.

Source of support: Nil

Conflict of interest: None

\section{INTRODUCTION}

Muscle tension dysphonia (MTD) is a condition where excessive muscular tension, in laryngeal and paralaryngeal areas, or muscle misuse, occur during phonation. Various synonyms have been used for this entity like hyperkinetic dysphonia, musculoskeletal tension dysphonia, hyperfunctional dysphonia, mechanical voice disorder, functional hypertensive dysphonia, muscle misuse dysphonia, laryngeal isometric dysphonia,

\footnotetext{
${ }^{1}$ Postgraduate, ${ }^{2}$ Assistant Professor

${ }^{1,2}$ Department of Otorhinolaryngology, Silchar Medical College and Hospital, Silchar, Assam, India

Corresponding Author: Sachender Pal Singh, Postgraduate Department of Otorhinolaryngology, Silchar Medical College and Hospital, Silchar, Assam, India, Phone: 07023733126 e-mail: sachender123@gmail.com
}

laryngeal tension fatigue syndrome. ${ }^{1}$ It has multifactorial etiologies. It can be primary or secondary MTD. The letter is due to compensatory behavior of phonation in diseases which affects either the aerodynamic configuration (like vocal fold paralysis), or the vibratory property of glottis (like vocal cord nodule). However, when the MTD is present without the anatomic or neurologic factors, then it is called as primary MTD. While it can affect anyone, sufferers usually belong to a particular group like teachers, singers and actors, frequent cell phone users and instructors, etc. who are likely to speak loudly, for long hours with inappropriate pitch or without following vocal hygiene. A detailed history and complete examination is necessary to diagnose MTD. It has very serious impact on sufferer's personal, social and professional life and significantly decreases the quality of life.

\section{MATERIALS AND METHODS}

This study is a prospective study during the period of June 2012 to January 2014 carried out at Department of ENT, at Silchar Medical College, Silchar, Assam.

\section{Subjects}

Twenty-seven subjects with MTD were selected for the study after making a proper diagnosis on the basis of history, clinical and laryngoscopic examination. The patients were in the age group of 20 to 70 years.

Patients included in the study were: Primary MTD (13), secondary MTD (14): vocal cord nodule (5), vocal cord polyp (2), cut throat injury (1), laryngopharyngeal reflux (4), presbylaryngis (2).

Patients excluded from the study were: Those who did not come for follow-up.

All the excised tissues of secondary MTD (vocal cord nodule and polyp) cases were sent for histopathological examination.

\section{Voice Outcome Measures}

The voice was recorded before and after voice therapy and voice outcome was based on auditory-perceptual ratings, quality of life measures and visual-perceptual ratings.

\section{Auditory-Perceptual Ratings}

Subjects were asked to read 'The Rainbow passage' (operating techniques in laryngology) or to count 1 to 20 and voice was recorded. Perceptual ratings of voice 
quality were conducted with the 'GRBAS scale'.13 The GRBAS scale is considered by many authors to be the most reliable auditory perceptual scale currently available for use as an outcome measure. ${ }^{2,3}$

\section{Quality of Life Measures}

'Voice handicap index' (VHI) was used to assess the impact of the voice in terms of physical complaint and restriction in participation in daily activities and response to treatment. ${ }^{4-8}$

\section{Visual-Perceptual Ratings}

It was based on comparison of Transnasal flexible Videolaryngoscopy (TFL) done before and after the voice therapy.

\section{RESULTS (GRAPHS 1 AND 2)}

Results of voice outcome measure are depicted in graph 1 (GRABS score) and graph 2 (VHI score) which shows that voice of the patients has improved a lot after the implication of voice therapy (Table 1).

\section{DISCUSSION}

Muscle tension dysphonia is one of the common voice disorders, with primary MTD accounting for approximately 10 to $40 \%$ of the caseload of a typical voice center (Roy, 2003).

In our study, we made the diagnosis on the basis of history, clinical and videolaryngoscopic examination. We found tenderness over different sites in the neck which were related to their respective excessive muscle tension. We started voice therapy before the surgery, in cases of vocal cord nodule/polyp so that their habits of voice abuse and misuse and compensatory behavior do not have worse affect postoperatively. Patients were informed about their diagnosis, anatomy and physiology of normal vocal tract in order to minimize the anxiety and stress and also to develop their belief in the voice therapy (Table 1) so that drop-out cases may be reduced. Vocal

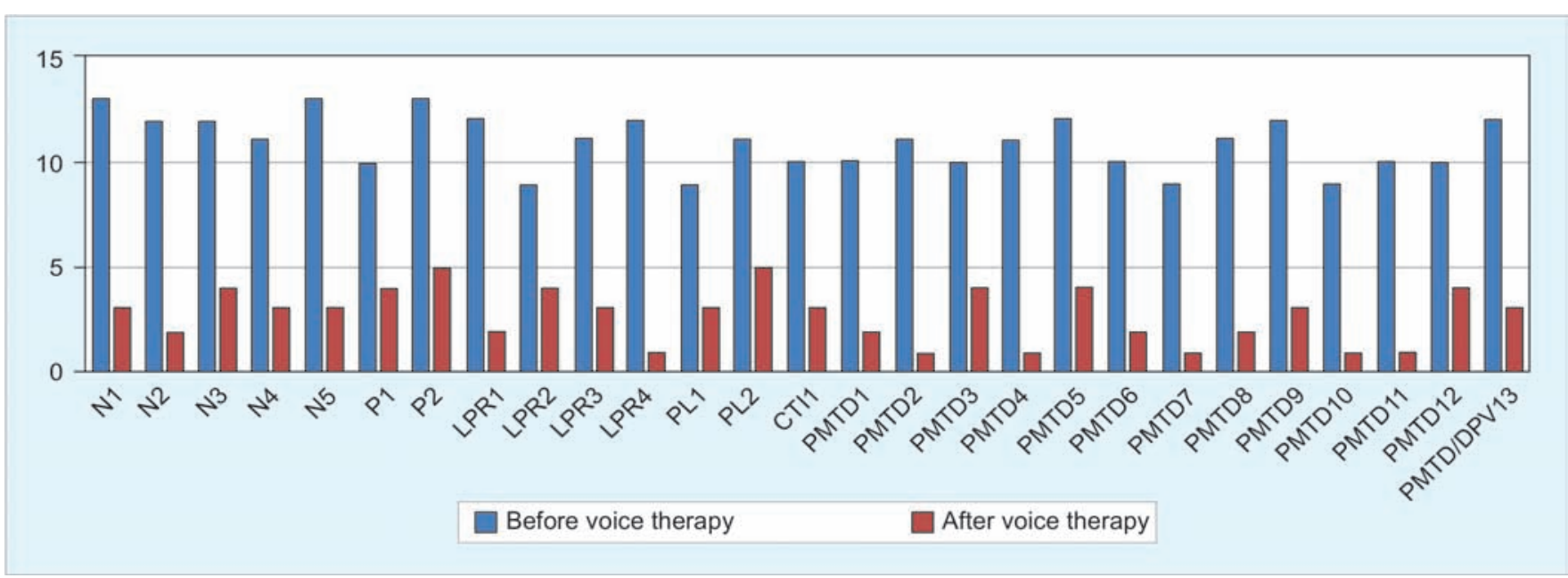

Graph 1: Grabs score

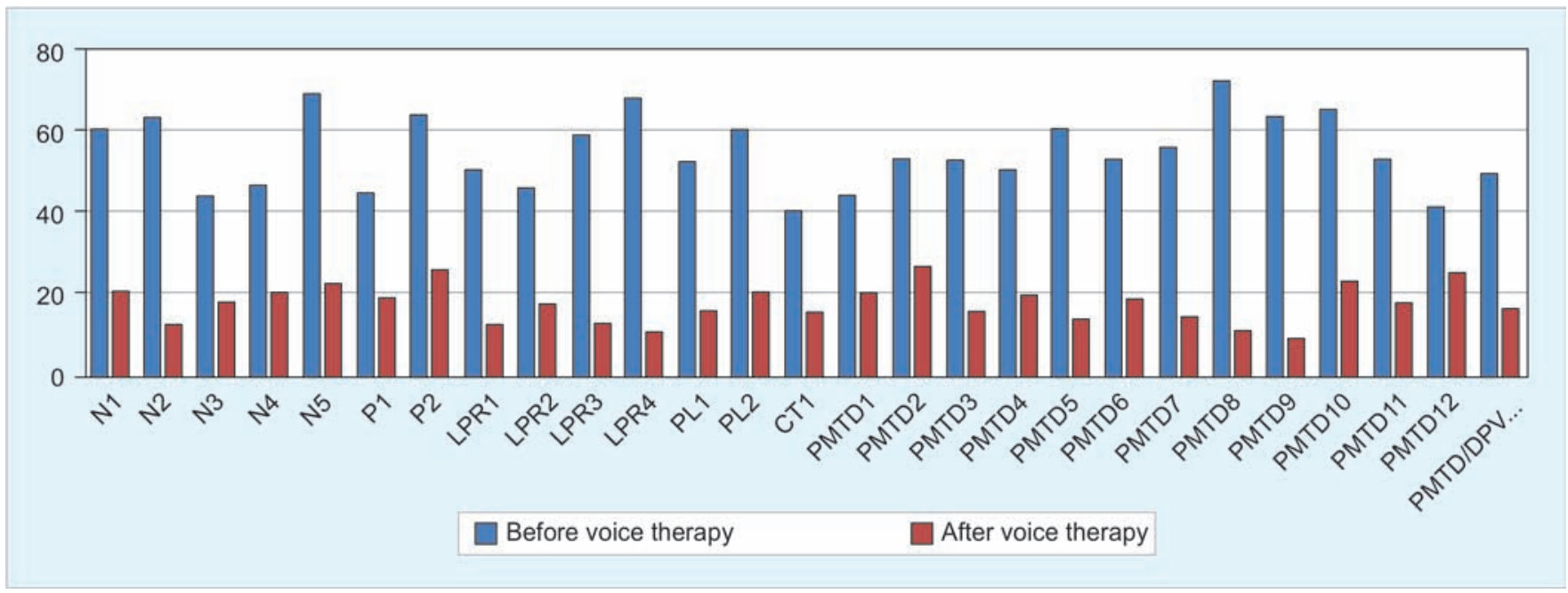

Graph 2: Voice handicap index score

(N: Vocal cord nodule; P: Vocal polyp; LPR: Laryngopharyngeal reflux; PL: Presbylaryngis; CT: Cut throat injury; PMTD: Primary muscle tension dysphonia; DPV: Dysphonia plica ventricular) 
Table 1: Voice therapy

- Vocal hygiene

- Symptomatic voice therapy: Circumlaryngeal massage, chewing exercises, yawn sigh approach, phonation on inhalation

- Respiratory retraining: Breath support, confidential voice therapy

- Physiologic voice therapy: Vocal function exercises, accent method, resonant voice therapy

- Psychogenic voice therapy

hygiene was instructed to every patient to eliminate the environmental and behavioral factors. Posture and mouth opening during phonation were corrected. Inappropriate muscle usage causing muscle tension at neck, floor of mouth or jaw were managed accordingly by massaging the muscle in tension, in the line of their muscle fibers. Treatment generally targets the increased hyolaryngeal elevation and laryngeal and perilaryngeal muscular tension. Circumlaryngeal massage was considered to treat extrinsic laryngeal muscle tension. Patients were considered for symptomatic, physiologic and psychogenic voice therapy in addition to respiratory retraining as and when required. In one of our cases, i.e. dysphonia plica ventricularis, we considered psychotherapy because he was having anxiety and depression due to failure in examination during the onset of symptoms. Another case, i.e. cut throat injury, was interesting. He presented with homicidal cut throat injury and was managed accordingly. But, during the course, he developed MTD, as a compensatory behavior. A bulge in the neck above the larynx could easily be appreciated during phonation. Then, after the end of primary treatment he was given voice therapy (Table 1). We have also considered psychotherapy in other primary MTD whenever we found it necessary on the basis of history. Associated laryngopharyngeal reflux was also treated as and when required. Results of voice therapy (Graphs 1 and 2) are satisfying to patient as well as to the trainer.

Symptoms in MTD are usually husky, hoarse, breathy and/or rough voice, decreased loudness, difficult or effortful phonation, deterioration of voice with prolonged use. Other symptoms may be present like irritation in throat, feeling tightness or a sensation of a lump in the throat, having to frequently clear the throat and increased mucus in the throat.

On examination, posture of body during phonation may be poor leading to increased laryngeal and paralaryngeal tension. Increased muscle tension hamper the movement of larynx on phonation. Patient may have developed tilting of neck while using mobile phone excessively. Due to increased tone in the thyrohyoid and tongue base muscles, there is elevation of the larynx and hyoid bone which stiffen the vocal folds 9 and produce anteroposterior supraglottic contraction ${ }^{10}$ affecting the phonatory patterns. Overuse of specific muscles will cause tenderness over that particular site; this can be elicited by slight pressure with forefingers. Muscle tension dysphonia patients are usually assessed by palpation for elevated laryngeal position, increased extrinsic laryngeal muscle activation ${ }^{10-15}$ shortening of the sternocleidomastoid and stylohyoid muscles. ${ }^{12}$ Muscle misuse and non muscle-misuse dysphonia can be differentiated with increased palpable tension (Angsuwarangsee and Morrison).

Auditory perceptual features of MTD include strained or effortful voice quality, aberrant pitch, breathiness or vocal fatigue. ${ }^{15,16}$

Physiologic features of MTD are elevated hypopharyngeal position, decreased space between hyoid and larynx and increased extrinsic laryngeal muscle tension. Hyolaryngeal elevation and excessive extrinsic laryngeal muscle activation can influence the mechanics of vocal fold vibration ${ }^{9,17}$ and are considered important contributors to the dysphonia in MTD.

Muscle tension dysphonia can be diagnosed on transnasal fiberoptic videolaryngoscopy on the basis of presence of anteroposterior squeezing with arytenoid and epiglottic apposition severly affecting vocal fold output, shortening of vocal folds with increase in mass and stiffness, adduction of false vocal cords with ventricle compression, increased adductor muscle tone or inappropriate vocal cord closure. ${ }^{18}$ Inappropriate closure of vocal folds can be appreciated as elliptical opening or bowing, hour glass shape, anterior chink, posterior chink, variable position of glottal opening, incomplete closure along most of the length of vocal cord.

There can be a diagnostic confusion between adductor spasmodic dysphonia and MTD due to voice characteristics that can mimic each other but, apart from the discussion of phonatory breaks and laryngeal airflow, effectively distinguish MTD from a neurologic disorder. ${ }^{16,19,20}$

Voice abuse and misuse lead to the development of vocal lesions (i.e. nodules), leading to altered phonatory behaviors to compensate the glottal insufficiency. This is expected to heighten the shearing forces at the site of a lesion, enhancing its maturity. ${ }^{21}$ This is a vicious cycle. Alternatively, development of MTD may have contributions from psychological and behavioral component which may lead to development of the vocal nodules, polypoidal degeneration or chronic laryngitis. ${ }^{22,23}$ Voice rest during acute laryngitis can prevent MTD by avoiding 
training of the sensorimotor system in the presence of altered feedback. In addition to all of the above, a surgery should be considered if needed in addressing etiology or the effect of MTD (like vocal cord nodule).

Management of MTD is multidisciplinary involving otorhinolaryngologist, psychotherapist and general physician. Interrelationship of the precipitating factors can be divided into four 'platforms':

Posture and muscle usage: An improper posture during phonation itself can lead to imbalance in the laryngeal musculature leading to MTD. Straight head, neck and back, relaxed shoulder (no drooping), correct breath support in relaxed or unstrained manner are necessary for proper phonation.

Behavioral and environmental factors: An understanding of the environmental and behavioral factors on phonation allows their application in management of MTD. Environmental factors, like dust, smoke, dry air, poor acoustics or amplifications, background noise and inadequate rest during phonation, should be corrected. Patient's faulty behavior, like throat clearing or coughing, dehydration, voice abuse and misuse, etc. should be corrected in order to treat MTD efficiently and effectively.

Laryngopharyngeal reflux: This is usually the cause of patient's faulty behavior and is also a predisposing factor for MTD. ${ }^{24}$ Management of this should be included in the management of MTD.

Psychological: Patient's anxiety, emotional distress and psychological status need to be addressed in order to manage MTD. Patient should be reassured about the disease; that there is no serious pathology and thorough explanation of anatomy and physiology of the vocal tract is needed. If there is any other psychological problem, it should be addressed accordingly with the help of psychotherapist. All these will help the patient to relax, be confident and also encourage him to continue the treatment properly.

\section{CONCLUSION}

Muscle tension dysphonia, whether primary or secondary, is due to imbalance in the laryngeal and paralaryngeal musculature during phonation caused by a diverse number of etiological factors viz. vocal misuse/abuse, psychological/personality disorders, compensatory vocal habits in case of upper airway infections, organic lesions and laryngopharyngeal reflux. Muscle tension dysphonia is diagnosed on the basis of detailed history, and clinical and videolaryngoscopic examinations. Every aspect of MTD whether postural, behavioral, environmental or psychological should be considered and managed appropriately. Laryngopharyngeal reflux should be treated appropriately. In cases of secondary MTD, the etiological factor, like benign vocal cord lesions should be managed surgically, if needed, to ameliorate the compensatory behavior. Vicious cycle of etiology and effect needs to be interfered as early as possible to stop the deterioration of voice further or to prevent the development of compensatory behavior. The findings (Graphs 1 and 2) suggest that voice therapy (Table 1) is an inevitable and efficient tool to manage MTD, and provide both objective and patient-centered outcomes.

\section{REFERENCES}

1. Altman KW, Atkinson C, Lazarus C. Current and emerging concepts in muscle tension dysphonia: a 30 months review. J Voice 2005;19(2):261-267.

2. Dejonckere P, Obbens C. Perceptual evaluation of dysphonia: reliability and relevance. Folia Phoniatr (Basel) 1993;45(2): 76-83.

3. DeBodt M, Wuyts F. Test-retest study of GRBAS scale: influence of experience and professional background on perceptual rating of voice quality. J Voice 1997;11(1):74-80.

4. Mathieson L. Voice disorders: presentation and classification. In: Mathieson L, editor. The voice and its disorders. London: Whurr Publishers; 2001. p. 121-144.

5. Carding PPN, Horseley IA. An evaluation study of voice therapy in non-organic dysphonia. Eur J Disorders of Communication 1992;27(2):137-158.

6. Scott S, Robinson K, Wilson JA, Mac Kenzie K. Patient reported problems associated with dysphonia. Clinical Otolaryngol 1997;22(1):37-40.

7. Hogikyan ND, Wodchis WP, Terrell JE, Bradford CR, Esclamado RM. Voice-related quality of life (V-RQOL) following type 1 thyroplasty for unilateral vocal fold paralysis. Journal of Voice 2000;14:378-386.

8. World Health Organization. Towards a common language for functioning and disablement: ICIDH-2. Geneva: WHO, 1998.

9. Shipp T. Vertical laryngeal position: research findings and applications for singers. J Voice 1987;1(3):217-219.

10. Angsuwarangsee T, Morrison M. Extrinsic laryngeal muscular tension in patients with voice disorders. J Voice 2002;16(3):333-343.

11. Aronson AE. Clinical Voice Disorders. 3rd ed. New York, NY: Thieme Stratton; 1990. p. 250-251.

12. Rubin JS, Blake E, Mathieson L. Musculoskeletal patterns in patients with voice disorders. J Voice 2007;21(4):477-484.

13. Roy N, Ford CN, Bless DM. Muscle tension dysphonia and spasmodic dysphonia: the role of manual laryngeal tension reduction in diagnosis and management. Ann Otol Rhinol Laryngol 1996;105(11):851-856.

14. Kooijman PG, de Jong FI, Oudes MJ, Huinck W, van Acht H, Graamans K. Muscular tension and body posture in relationto voice handicap and voice quality in teachers with persistent voice complaints. Folia Phoniatr Logop 2005;57(3):134-147.

15. Altman KW, Atkinson C, Lazarus C. Current and emerging concepts in muscle tension dysphonia: a 30-month review. J Voice 2005;19(2):261-267. 
16. Roy N, Whitchurch M, Merrill RM, Houtz D, Smith ME. Differential diagnosis of adductor spasmodic dysphonia and muscle tension dysphonia using phonatory break analysis. Laryngoscope 2008;118(12):2245-2253.

17. Sundberg J, Askenfet A. Larynx height and voice source: a relationship? In: Bless DM, Abbs JH, editors. Vocal Fold Physiology: Contemporary Research and Clinical Issues. San Diego, CA: College Hill Press; 1983. p. 307-316.

18. Muscle tension dysphonia, 'Voice' 1997. The J Australian Voice Association. Retrieved from https://www.google. co.in $/$ url?sa $=$ t\&rct $=j \& q=\& e s r c=s \&$ source $=$ web $\& c d=1 \& c a$ $\mathrm{d}=$ rja\&uact $=8 \&$ ved $=0 \mathrm{CCMQFjAA \& url}=\mathrm{http} \% 3 \mathrm{~A} \% 2 \mathrm{~F} \% 2 \mathrm{~F}$ voiceconnection.com.au\%2Fpdf\%2Fmuscletension.pdf\& ei=4cCTVZe2BMqgugSc6IOwCQ\&usg=AFQjCNF4uHqf FgCN64y3pakmyRm54gLtmQ\&sig2=eSD0FAuewKHE8_ STmEYUWA\&bvm=bv.96952980,d.c2E

19. Sapienza CM, Walton S, Murry T. Adductor spasmodic dysphonia and muscular tension dysphonia: acoustic analysis of sustained phonation and reading. J Voice 2000;14(4): 502-520.

20. Higgins MB, Chait DH, Schulte L. Phonatory air flow characteristics of adductor spasmodic dysphonia and muscle tension dysphonia. J Speech Lang Hear Res 1999;42(1): 101-111.

21. Jiang J, Diaz C, Hanson D. Finite element modeling of vocal fold vibration in normal phonation and hyperfunctional dysphonia: implications for the pathogenesis of vocal nodules. Ann Otol Rhinol Laryngol 1998;107(7):603-610.

22. Aronson A. Clinical Voice Disorders: An Interdisciplinary Approach. New York: Thieme Inc; 1985 p. 161-183.

23. Colton R, Casper J. Understanding Voice Problems: A physiological perspective for diagnosis and treatment. Baltimore, MD: Williams and Wilkins; 1990. p. 107-149.

24. Koufman J, Amin M, Panetti M. Prevalence of reflux in 113 consecutive patients with laryngeal and voice disorders. Otolaryngol Head Neck Surg 2000;123(4):385-388. 